\title{
Effects of Mask Use and Other-Race on Face Perception, Emotion Recognition, and Social Distancing During the COVID-19 Pandemic
}

Evrim Gulbetekin ( $\square$ evrimg@akdeniz.edu.tr)

Akdeniz University

Arda Fidancı

Cognitive Science Department, University of Minessota, USA

Enes Altun

Akdeniz University, Department of Psychology

Muhammed Nurullah Er

Akdeniz University, Department of Psychology

\section{Esin Gürcan}

Akdeniz University, Department of Psychology

\section{Research Article}

Keywords: face recognition, mask use, facial expressions, social distance

Posted Date: August 16th, 2022

DOI: https://doi.org/10.21203/rs.3.rs-692591/v2

License: (9) This work is licensed under a Creative Commons Attribution 4.0 International License. Read Full License 


\section{Abstract}

We tested the effect of mask use and other-race effect (ORE) on (a)face recognition, (b)recognition of facial expressions, and (c)social distance. Caucasian subjects were tested in a matching-to-sample paradigm with either masked or unmasked Caucasian and Asian faces. The participants exhibited the best performance in recognizing an unmasked face condition and the poorest to recognize a masked face that they had seen earlier without mask. Accuracy was poorer for Asian faces than Caucasian faces. The second experiment presented Asian or Caucasian faces having emotional expressions, with and without masks. The participants emotion recognition performance decreased for masked faces. From the most accurately to least accurately recognized emotions were as follows: happy, neutral, disgusted, fearful. Performance was poorer for Asian stimuli compared to Caucasian. In Experiment 3 the same participants indicated the social distance they would prefer with each pictured person. They preferred a wider distance with unmasked faces compared to masked faces. Distance from farther to closer was as follows: disgusted, fearful, neutral, happy. They preferred wider social distance for Asian compared to Caucasian faces. Altogether, findings indicated that during the COVID-19 pandemic mask wearing decreased recognition of faces and emotional expressions, negatively impacting communication among people from different ethnicities.

This investigation used three experiments to test the effect of mask use and other-race effect (ORE) on face perception in three contexts: (a) face recognition, (b) recognition of facial expressions, and (c) social distance. The first, which involved a matching-to-sample paradigm, tested Caucasian subjects with either masked or unmasked faces using Caucasian and Asian samples. The participants exhibited the best performance in recognizing an unmasked face condition and the poorest when asked to recognize a masked face that they had seen earlier without a mask. Accuracy was also poorer for Asian faces than Caucasian faces. The second experiment presented Asian or Caucasian faces having different emotional expressions, with and without masks. The results for this task, which involved identifying which emotional expression the participants had seen on the presented face, indicated that emotion recognition performance decreased for faces portrayed with masks. The emotional expressions ranged from the most accurately to least accurately recognized as follows: happy, neutral, disgusted, and fearful. Emotion recognition performance was poorer for Asian stimuli compared to Caucasian. Experiment 3 used the same participants and stimuli and asked participants to indicate the social distance they would prefer to observe with each pictured person. The participants preferred a wider social distance with unmasked faces compared to masked faces. Social distance also varied by the portrayed emotion: ranging from farther to closer as follows: disgusted, fearful, neutral, and happy. Race was also a factor; participants preferred wider social distance for Asian compared to Caucasian faces. Altogether, our findings indicated that during the COVID-19 pandemic mask wearing decreased both face recognition and recognition of emotional expressions, negatively impacting communication among people from different ethnicities.

\section{Background}


The face provides a rich source of information about its owner's gender, race, age, physical health, attractiveness, and emotional state. However, following the spread of the COVID-19 pandemic throughout the globe, humans' daily routines have changed, involving three major precautions: face-mask wearing, hand washing, and social distancing. Two of these precautions-using face masks and maintaining social distance-affect individuals' perceptions of faces due to the associated restriction of their visual field.

Masks cover specific areas of the face, including the nose, mouth, chin, and most of the cheeks. Before the current pandemic, prior research had focused on the impairing effects of facial parts occlusion in the context of face recognition and recognition of emotional expressions. For example, Bassili (1979) demonstrated negative effects resulting from the partial occlusion of the face using a cardboard rectangle. According to the researcher's findings, occlusion of the "lower-facial" part negatively affected participants' ability to recognize happiness and sadness. In contrast, recognition of anger, which mostly relies on the eye region, was less affected. Another study (Roberson et al., 2012) using sunglasses and masks as partial occluders showed that occlusion decreased emotion recognition performance. In a similar vein, Dhamecha et al. (2014) indicated that hiding facial parts with accessories such as glasses and masks impaired face recognition ability since they reduced subjects' uniqueness. The authors also found recognition accuracy was better for faces from the same ethnicity of the participants. Other studies investigated the effects of covering the face and head in the context of the Islamic headdress (Fischer et al., 2012; Megreya \& Bindemann, 2009; Toseeb et al., 2014) Headscarves which covers external features such as hair did not influence face recognition dramatically while Fisher et al. (2012) shown that wearing niqab which covers some internal facial areas such as mouth and nose impaired positive emotion recognition but not negative emotion recognition. Kret and De Gelder (2012) indicated that although the expressions of fear and anger were still recognizable in the niqab and two different burqa conditions ( $20 \%$ and $10 \%$ transparency conditions), reduced accuracy was observed for happy and sad expressions in all of these conditions. A recent study, researchers (Noyes et al., 2021) investigated the effects of masks and sunglasses on familiar and unfamiliar face-matching and emotion categorization performance in super-recognizers and control subjects. The researchers found that although superrecognizers' accuracy in performance was higher than that of the controls, both masks and sunglasses reduced subjects' performance in all of the experimental tasks. A more recent study (Blazhenkova et al., 2022) indicated that face masks either having curved/angular pattern or different colors decreased emotion recognition. On the other hand, it seems that transparent masks do not distort emotion recognition (Marini et al.,2021).

Multiple studies have shown that an individual's face recognition system depends on holistic processing rather than partial characteristics of faces (Farah et al., 1998; Tanaka \& Farah, 1993; Yin, 1969; Young et al., 1987). More recently, Freud et al. (2020) showed that face recognition performance and holistic face processing decreased when participants were exposed to faces with masks during the COVID-19 pandemic. Another recent study (Carragher \& Hancock, 2020) found that surgical face masks decreased human face matching performance, and the impairment is similar for both familiar and unfamiliar faces. While Carbon (2020) focused on the effects of face masks on emotion recognition and indicated 
that although face masks reduced participants' recognition accuracy for some emotions (angry, disgusted, happy, and sad), neutral and fearful faces were still recognizable when masked.

Although previous studies have shown that mask use decreases the recognition of faces and emotional expressions, how mask use affects individuals' perceptions of faces from other races remains unclear. In 2001, Meissner and Brigham defined "own-race bias" (ORB) as humans' ability to recognize own-race faces better than other-race faces. ORB has also been shown to affect the holistic face processing system (Michel et al., 2006; Tanaka et al., 2004). Since people perceive faces holistically, facial identification is remarkably more efficient for faces that share the same environment as the viewer while less powerful regarding the faces of other ethnicities that viewers have less experience with (Hancock \& Rhodes, 2008; Tanaka et al., 2004). Moreover, some studies have shown that exposure to the faces of people from other ethnic groups plays a critical role in facial recognition for other-race faces. For example, Heron-Delaney et al. (2011) demonstrated that increased exposure to faces belonging to other races could reduce the other-race effect in infants. On another note, Cavazos et al. (2018) observed the interaction effects of expertise and socio-cognitive factors in their study, finding that Caucasian participants demonstrated own-race advantages in a multi-image learning task, while East Asian participants showed enhanced learning for other-race identities during a matching task. Dal Martello and Maloney (2006) showed that upper face side (mostly eye region) contributed kin recognition more than the lower face side. To our knowledge, no existing study has investigated whether mask use affects ORB. Accordingly, the current study examines other-race effects in processing faces with masks in addition to face recognition and recognition of emotional expressions.

Social distancing after the COVID-19 pandemic is the second factor that this study considers. In humans, detecting the identity of another person and interacting usually begin with face perception. People can quickly identify whether a person is familiar or unfamiliar or a threat or not due to humans' superior face recognition ability. Thus, individuals may tend to remain close to someone or keep their distance from others depending on their perception of others as friends or threats. In one prior study, after collecting data from 42 countries, Sorokowska et al. (2017) showed that average interpersonal distance varied based on the type of distance, with an average of $135.1 \mathrm{~cm}$ for social distance. The participants' gender and country's average temperature also served as factors that predicted the preferred social distance. The researchers' results revealed that people prefer to be closer to strangers in warmer countries while preferring to be farther from their partners. Nevertheless, the emergence of the COVID-19 pandemic caused people to readjust their preferred interpersonal distances to avoid exposure to the virus. Although health authorities have suggested that social distancing is a pro-social behavior in response to COVID-19, involving separation of at least 1.5-2 meters (Chu et al., 2020), some researchers have noted that this minimum distance conflicts with people's norms (Welsch et al., 2020).

Since the emergence of COVID-19, masked faces have come to be associated with the "pandemic" situation. Hence, face masks may be considered an essential signal for adjusting social distance with others. Until the COVID-19 pandemic, only people who had particular medical reasons wore face-covering masks in public. Therefore, at the beginning of the COVID-19 pandemic, wearing facemasks might have 
led to a negative perception of mask wearers. However, once health authorities informed the public that facial masks increased the protection against COVID-19, mask use became more prevalent, potentially leading to a more positive perception of individuals wearing masks.

In summary, facial masks may pose a threat or a safety signal to adjust social distance with other people. One recent study demonstrated that wearing a facial mask reduced social distancing (Cartaud et al., 2020). In that study, the participants' perceptions toward people wearing facial masks changed as they considered the latter safer than non-mask wearers. Another variable that masks might affect and that might modulate social distancing is the perception of risk. For example, a recent study conducted with Chinese residents demonstrated that social distancing behavior significantly increased with increasing perceived risk (Xie et al., 2020). On the other hand, emotional expression studies have demonstrated that behaviors that are linked to social distance depend on the ability to perceive other people's facial expressions. For instance, one study demonstrated that perceiving positive emotions (such as happiness) rather than negative emotions (e.g., anger) led to a reduced social distance toward other people (Ruggiero et al., 2017). Since a mask covers part of the wearer's face, mask wearing potentially detracts from recognizing emotional expressions. Presumably, people seek to adjust their social distance to avoid virus exposure, a process that may also be influenced by mask use, emotional expressions, and the race of the other person.

In light of these conditions during the COVID-19 pandemic, we conducted three experiments with Caucasian participants to test the effect on face perception of mask use and other-race effect in three contexts: (a) how mask use influences face recognition, (b) how mask use influences the recognition of facial expressions, and (c) how mask use interacts with the facial expression and race of the other person to influence social distance. The first experiment used a matching to sample paradigm. Masked or unmasked faces from the same or different races were presented as samples; the subjects were tested with both masked or unmasked faces. In the second experiment, faces from the same or different races with the participants and with and without masks were presented having different emotional expressions, and the participants were asked to identify which emotional expression they saw. Since sad, fearful and angry faces are most often recognized by upper facial parts, while happiness and disgust are recognized by information in the lower facial part (Wegrzyn et al., 2017), we expected to observe different mask effects on different emotional expressions. In Experiment 3, following the second experiment, the same faces with and without masks were shown individually, and the participants in Experiment 2 were asked to indicate the social distance they preferred to maintain with that person.

\section{Experiment 1}

\section{Method}

\section{Participants}

A total of 102 undergraduate students (80 females and 22 males) with a mean age of 20.4 years $(M=$ $20.4, S D=2.8$ ) participated in the study. They had no neurological or psychological disorders, nor were 
they receiving any medical treatment at the time of the research, according to their self-report.

The study was approved by the Akdeniz University Ethical Committee for Social Sciences. This study's design and hypotheses were preregistered; see [https://osf.io/wxpr4/]. The data was collected between the dates of June and September 2020.

\section{Procedure}

\section{Stimuli}

Twenty-four Asian and 24 Caucasian facial stimuli ( 24 female and 24 male) were used from the racially diverse affective expression (RADIATE) face stimulus set (Conley et al., 2018) and the MR2 (a multi-racial mega-resolution database of facial stimuli; Strohminger, 2016). We used neutral faces in Experiment 1. All stimuli were processed using the SHINE toolbox in MATLAB to control luminance and spatial frequency (Dal Ben, 2019). Facial photographs were cropped under the chin region, and a white background was used.

Surgical face mask images were superimposed on all of the facial stimuli using Adobe Photoshop software to create faces with masks. The same surgical face mask image was used for all the masked stimuli. The mask was positioned to cover each image's chin, nose, and most of the cheek area. We did not cover the hair to ensure external validity of facial stimuli. Figure 1 provides two examples of samples with and without the mask.

\section{Experimental Procedure}

Experiment 1 involved a matching to sample procedure. A face without a mask was presented, followed by two faces (including the target face). The participants were asked to choose the face that had been presented as a sample. The experimental sessions incorporated four conditions:

1. The sample was an unmasked face; the test faces were also unmasked.

2. The sample face was masked; the test faces were also masked.

3. The sample face was unmasked, but the test faces were masked.

4. The sample face was masked, but the test faces were unmasked.

Figure 2 illustrates all four experimental conditions. Nine practice trials were conducted before the original experimental trials. The participants received feedback in the practice trials. A total of 80 experimental trials were conducted. The stimuli were presented in a pseudo-random order.

A fixation cross was presented for $500 \mathrm{~ms}$. Next, the sample face was presented for $500 \mathrm{~ms}$. Following the sample face, two faces were presented for $800 \mathrm{~ms}$, and the participants were asked to decide which face was the sample. The participants pressed " 1 " on the keyboard to choose the stimulus on the left side of the screen and " 0 " to choose the stimulus on the right side of the screen. No feedback was given in the experimental trials. Figure 3 illustrates the experimental procedure. 
Due to the pandemic conditions, data collection employed an online platform. Fifty participants were tested using the online experiment platform Testable, and 52 participants were tested via E-Prime Go. The same procedures were applied on both platforms. Participants' responses and accuracy were recorded.

\section{Results}

\section{Accuracy}

A $4 \times 2$ (mask conditions [(1) unmasked sample-unmasked test, (2) masked sample-masked test, (3) unmasked sample-masked test, (4) masked sample-unmasked test] $\times$ race [Asian, Caucasian]) repeated ANOVA was conducted to determine the effects of mask conditions and race of facial stimuli on discrimination accuracy. Since the sphericity assumption was violated, the Greenhouse-Geisser correction was applied. The results indicated a significant main effect of race $F(1,101)=90.28, p=.001$ $\eta \mathrm{p} 2=.47$. Accuracy for Caucasian faces $(n=102, M=.96, S D=.08)$ was higher than for Asian faces $(n=$ $102, M=.92, S D=.09)$. The mask condition also had a significant effect on accuracy $F(2.6,263.08)=$ $60.79, p=.001 \mathrm{np} 2=.38$. The participants demonstrated the highest performance when they were shown an unmasked sample and tested with unmasked faces. In comparison, they produced the worst performance when shown an unmasked sample and tested with masked faces. Table 1 presents descriptive statistics for mask conditions. Bonferroni pairwise comparison results indicated that all the differences among the conditions were significant $(p<.05)$; however, the difference between masked sample-masked test and masked sample-unmasked test was not significant $(p>.05)$. The results of Bonferroni pairwise test can be found in supplementary materials.

\section{Table 1}

Descriptive Statistics (Means and Standard Deviations) for Accuracy in Respect to Mask Conditions

\begin{tabular}{lcc} 
& $M$ & $S D$ \\
\hline Unmasked sample-Unmasked test & .97 & .09 \\
\hline Unmasked sample-Masked test & .89 & .07 \\
\hline Masked sample-Unmasked test & .94 & .09 \\
\hline Masked sample-Masked test & .95 & .09
\end{tabular}

We also found a significant interaction effect of race and mask conditions $F(2.61,263.50)=37.84, p=$ $.001, \mathrm{np} 2=.27$. Therefore, two repeated measures ANOVAs were conducted to determine the effects of mask conditions for Asian and Caucasian faces separately. Since the sphericity assumption was violated, the Greenhouse-Geisser corrections were applied in these analyses. Within-subject factor results for Asian faces indicated a significant main effect of the mask condition $F(1.02,278.16)=76.62, p=$ $.001, \mathrm{np} 2=.43$. The participants yielded the highest performance when they were shown an unmasked 
sample and tested with unmasked faces $(p<.05)$. Meanwhile, they demonstrated the worst performance when they were shown an unmasked sample and tested with masked faces $(p<.05)$. Within-subject factor results for Caucasian faces also indicated a significant main effect of the mask condition $F(2.34$, $236.79)=4.405, p=.001, \mathrm{np} 2=.04$. Pairwise comparison tests indicated that the participants performed better in the unmasked sample-unmasked test condition than they did in all the other conditions $(p<$ .05). However, the performance difference among the other conditions was not significantly different for Caucasian stimuli $(p>.05)$. Table 2 presents descriptive statistics for Asian and Caucasian faces. The results of Bonferroni pairwise test can be found in supplementary materials.

\section{Table 2}

Descriptive Statistics [Mean (SD)] for Accuracy with Respect to Race and Mask Conditions

\begin{tabular}{lll} 
& Asian & Caucasian \\
\hline Unmasked sample-Unmasked test & $0.95(.11)$ & $0.98(.08)$ \\
\hline Unmasked sample-Masked test & $0.83(.11)$ & $0.96(.08)$ \\
\hline Masked sample-Unmasked test & $0.93(.09)$ & $0.95(.09)$ \\
\hline Masked sample-Masked test & $0.94(.11)$ & $0.96(.01)$
\end{tabular}

\section{Experiment 2}

\section{Method}

\section{Participants}

A total of 134 undergraduate students (105 females and 29 males) with a mean age of 21 years ( $M=$ $21.3, S D=1.6)$ participated in the study. They had no neurological or psychological disorders, nor were they receiving any medical treatment at the time of the research, according to their self-report. All participants were tested using the online experiment platform Testable.

The study was approved by Akdeniz University Ethical Committee for Social Sciences. This study's design and hypotheses were preregistered; see [https://osf.io/cwvup/]. The data was collected between the dates of June and September 2020.

\section{Experimental Procedure}

\section{Stimuli}

Facial stimuli were chosen from the racially diverse affective expression (RADIATE) face stimulus set. In total, we used eight Asian (four female, four male) and eight Caucasian (four female, four male) faces. Four emotional expressions (neutral, happy, fear, and disgust) were used among the 16 faces. Since our 
aim was to test the effect of mask use on emotion recognition, surgical face mask images were superimposed on all facial stimuli using Adobe Photoshop software, as in Experiment 1. The same surgical face mask image was used for all the stimuli. In total, we had 128 experimental stimuli: 64 unmasked faces and 64 masked versions of those faces.

A fixation cross was presented for 500 ms. Next, a neutral face or a face with an emotional expression (happy, fear, disgust) was presented on the center of the screen for 1,000 ms. The participants were asked to indicate which expression appeared on the presented face. Seven options (Happy, Sad, Surprised, Neutral, Fearful, Disgusted, Angry) were presented to choose. Six universal expressions and one neutral expression were represented. Figure 5 illustrates the experimental procedure.

Eight practice trials were conducted before starting the test trials. The experiment consisted of 128 trials in total. The stimuli were presented in pseudo-random order. No feedback was given in the experimental trials. Response accuracy was measured as the dependent variable.

All of the participants were tested using the online experiment platform Testable between the dates of June and August 2020.

\section{Results}

\section{Accuracy}

A $2 \times 2 \times 4$ (mask conditions [unmasked, masked] $\times$ race [Asian, Caucasian] $\times$ emotion [neutral, happy, fear, disgust]) repeated ANOVA was conducted to determine the effects of mask wearing, emotions, and race of facial stimuli on discrimination accuracy. Since the sphericity assumption was violated, the Greenhouse-Geisser correction was applied in the required analysis. The results indicated a significant

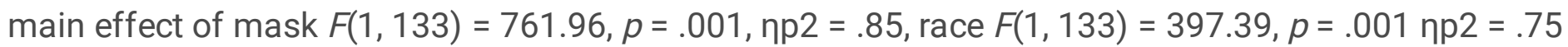
and emotion $F(1,363.12)=201.09, p=.001 \eta p 2=.60$. Expressions were better recognized on unmasked faces $(n=134, M=.75, S D=.08)$. than masked faces $(n=134, M=.55, S D=.09)$. They were also recognized better on Caucasian faces $(n=134, M=.71, S D=.09)$ than they were on Asian faces $(n=134$, $M=.58, S D=.08)$. The expressions from the best recognized to the least recognized were happy $(n=134$, $M=.84, S D=.16)$, neutral $(n=134, M=.73, S D=.15)$, disgust $(n=134, M=.56, S D=.10)$ and fear $(n=134$, $M=.45, S D=.15)$ respectively. Descriptive statistics were presented in Table 3.

\section{Table 3}

Descriptive Statistics [Mean (SD)] for Discrimination Accuracy Related to Emotion, Mask Condition, and Race 


\begin{tabular}{lllll} 
& Neutral & Happy & Fear & Disgust \\
\hline UM Asian & $0.72(.30)$ & $0.92(.17)$ & $0.41(.20)$ & $0.72(15)$ \\
\hline M Asian & $0.71(.19)$ & $0.66(.20)$ & $0.29(.20)$ & $0.22(.18)$ \\
\hline UM Caucasian & $0.77(.19)$ & $0.95(.17)$ & $0.58(.19)$ & $0.90(.11)$ \\
\hline M Caucasian & $0.72(.14)$ & $0.81(.21)$ & $0.54(.22)$ & $0.41(.19)$
\end{tabular}

The interaction of mask and race $F(1,363.12)=16.72, p=.001 \mathrm{np} 2=.11$, the interaction of mask and emotion $F(2.94,391.34)=213.53, p=.001 \mathrm{np} 2=.61$ and the interaction of race and emotion $F(2.51$, $334.028)=43.47, p=.001 \mathrm{np} 2=.25$ were statistically significant. In addition, the interaction of mask, race and emotion was also significant $F(2.64,351.071)=9.75, p=.001 \eta p 2=.07$.

Based on our observation of an interaction effect of race and emotion on discrimination accuracy, we conducted paired t-tests for each emotional expression. The results indicated that accuracy for each expression was higher for Caucasian faces in comparison to Asian faces, neutral $t(133)=-2.04, p=.04$; happiness $t(133)=-6,50, p=.001$; fear $t(133)=-15,36, p=.001$; disgust $t(133)=-18.09, p=.001$ (Table 4).

\section{Table 4}

Descriptive Statistics [Mean (SD)] for Accuracy Related to Emotion and Race

\begin{tabular}{|lllll|}
\hline & Happy & Neutral & Disgust & Fear \\
\hline Asian & $.79(.16)$ & $.71(.21)$ & $.47(.12)$ & $.34(.16)$ \\
\hline Caucasian & $.83(.17)$ & $.75(.14)$ & $.66(.12)$ & $.56(.18)$ \\
\hline
\end{tabular}

Based on the interaction effect of emotion and mask wearing on accuracy, we conducted paired $t$-tests to compare recognition performance for masked and unmasked faces. The results indicated that accuracy for each expression decreased when seen with masks, neutral $t(133)=1.94, p=.05$; happiness $t(133)=$ 15.29, $p=.001$; fear $t(133)=6.15, p=.001$; disgust $t(133)=15.29, p=.001$ (Table 5). The difference between accuracy for masked and unmasked neutral expressions was marginally significant. On the other hand, accuracy for expression of disgust decreased dramatically when the faces were masked.

\section{Table 5}

Descriptive Statistics [Mean (SD)] for Accuracy Regarding to Emotion, and Mask Condition 


\begin{tabular}{lcclc} 
& Neutral & Happy & Fear & Disgust \\
\hline Unmasked & $.74(.20)$ & $.94(.16)$ & $.49(.16)$ & $.81(.11)$ \\
\hline Masked & $.72(14)$ & $.73(.19)$ & $.41(.18)$ & $.32(.16)$
\end{tabular}

Upon observing the significant interaction of race, emotion, and mask wearing, we conducted paired ttests to compare each masked and unmasked condition for each race and emotion (Table 6). The best recognized expressions were unmasked Caucasian faces in all conditions. Although happiness was the most accurately recognized expression among all expressions for both unmasked Caucasian and Asian faces, performance decreased when the masked happy face was Asian. Fear was the least accurately recognized expression on both Caucasian and Asian faces. Interestingly, the participants could not recognize fear on Asian faces even when presented without a mask. Moreover, although disgust could be easily recognized on unmasked faces, recognition performance decreased when the faces were masked.

\section{Table 6}

Results of Paired t-tests for Masked and Unmasked Asian and Caucasian Faces

\begin{tabular}{|lllll|}
\hline Emotion & $\begin{array}{l}\text { Conditions } \\
\text { compared }\end{array}$ & Paired $t$ test results & Conditions & Paired $t$ test results \\
Neutral & Asian M-UM & $p>.05$ & $\begin{array}{l}\text { Caucasian M- } \\
\text { UM }\end{array}$ & $t(133)=2.97, p=.004$ \\
Happy & Asian M-UM & $\begin{array}{l}t(133)=17.01, p= \\
.001\end{array}$ & $\begin{array}{l}\text { Caucasian M- } \\
\text { UM }\end{array}$ & $\begin{array}{l}t(133)=-2.65, p= \\
.001\end{array}$ \\
\cline { 3 - 4 } & Asian M-UM & $\begin{array}{l}t(133)=6.19, p= \\
.001\end{array}$ & $\begin{array}{l}\text { Caucasian M- } \\
\text { UM }\end{array}$ & $t(133)=2.97, p=.001$ \\
Disgust & Asian M-UM & $\begin{array}{l}t(133)=25.26, p= \\
.001\end{array}$ & $\begin{array}{l}\text { Caucasian M- } \\
\text { UM }\end{array}$ & $\begin{array}{l}t(133)=-28.11, p= \\
.001\end{array}$ \\
\hline
\end{tabular}

\section{Experiment 3}

\section{Method}

\section{Participants}

The participants in Experiment 2 were also included in Experiment 3 to find out if there was any relationship between recognition of facial expression and social distance. They were tested using the online experiment platform Testable.

The study was approved by Akdeniz University Ethical Committee for Social Sciences.

\section{Experimental Procedure}




\section{Stimuli}

The same facial stimuli from Experiment 2 were used in Experiment 3. In all, eight Asian (four female, four male) and eight Caucasian (four female, four male) faces, four emotional expressions (neutral, happy, fear, and disgust), and mask conditions (masked and unmasked) were manipulated within 16 faces. A total of 128 stimuli (64 unmasked faces and 64 masked faces) were used in this experiment.

A fixation cross was presented for $500 \mathrm{~ms}$. Next, a neutral face or a face with an emotional expression (happy, fear, disgust) was presented on the center of the screen. The participants were asked to use a slider to indicate the social distance that they would prefer to keep with that person (0-8 meters). Figure 7 illustrates the experimental procedure.

\section{Results}

\section{Social Distance}

A $2 \times 2 \times 4$ (mask conditions [unmasked, masked] $\times$ race [Asian, Caucasian] $\times$ emotion [neutral, happy, fear, disgust]) repeated measures ANOVA was conducted to determine the effects of mask conditions, emotions, and race of the facial stimuli on social distance. Since the sphericity assumption was violated, the Greenhouse-Geisser correction was applied. The results indicated a significant main effect of mask wearing $F(1,133)=67.551, p=.001, \eta p 2=.34$ and emotion $F(1.48,196.175)=111.83, p=.001, \eta p 2=$ .46. The participants tended to indicate a preference for a wider social distance from unmasked faces ( $n$ $=134, M=4.47, S D=1.72)$ in comparison to masked faces $(n=134, M=3.62, S D=1.49)$. In addition, they tended to prefer greater social distances to faces having an expression of disgust $(n=134, M=4.66, S D=$ 1.59), fear $(n=134, M=4.52, S D=1.56)$, neutrality $(n=134, M=3.63, S D=1.67)$ and happiness $(n=134$, $M=3.37, S D=1.66)$ respectively. On the other hand, the main effect of race was not significant, $p>.05$.

The interaction of mask and emotion $F(2.17,288.72)=46.28, p=.001 \eta p 2=.26$, the interaction of mask and race $F(1,133)=42.19, p=.001 \mathrm{np} 2=.24$, and the interaction of race and emotion $F(2.27,301.91)=$ $6.89, p=.001 \mathrm{np} 2=.05$ were statistically significant. In addition, the interaction of mask, race and emotion was also significant $F(1,42,188,65)=33,72, p=.001$ np2 $=.20$.

After noting the significant interaction of mask and emotion, we conducted four paired $t$-tests to discover the effect of mask wearing on social distance for each emotion. The $t$-test results indicated a mask effect for neutral, $t(133)=4,18, p=.001$; happy, $t(133)=5.28, p=.001$; fear, $t(133)=9.02, p=.001$, and disgust expressions, $t(133)=10,46, p=.001$. The mask condition main effect on social distance was evident for all emotional expressions (Table 7). The participants tended to indicate a preference to remain far from unmasked faces. As already observed in the discussion of the emotion's main effect on social distance, the participants preferred to maintain the maximum distance from fearful and disgusted faces.

\section{Table 7}


Descriptive Statistics [Mean (SD)] for Social Distance with Respect to Masked and Unmasked Faces for Different Emotional Expressions

\begin{tabular}{lllll} 
& Neutral & Happy & Fear & Disgust \\
\hline Unmasked & $3.94(1.95)$ & $3.71(2.12)$ & $5.03(1.76)$ & $5.31(1.79)$ \\
\hline Masked & $3.37(1.52)$ & $2.89(1.54)$ & $3.99(1.63)$ & $4.02(1.68)$
\end{tabular}

Following the significant interaction of mask and race, we conducted two paired $t$-tests to discover the effect of race on social distance for each mask condition. The preferred social distance from Asian faces was wider for unmasked faces $t(133)=4,25, p=.001$, while the participants indicated a preference for a wider separation from Caucasian masked faces $t(133)=-2.65, p=.009$ (Table 8).

\section{Table 8}

Descriptive Statistics [Mean (SD)] for Social Distance with Respect to Masked and Unmasked Faces for Different Emotional Expressions

\begin{tabular}{lll} 
& Asian & Caucasian \\
\hline Unmasked & $4.56(1.79)$ & $4.37(1.68)$ \\
\hline Masked & $3.55(1.61)$ & $3.70(1.44)$
\end{tabular}

After noting the significant interaction of race and emotion, we conducted four paired $t$-tests to discover the effect of race on social distance for each emotion. The $t$-test results indicated a race difference for neutral, $t(133)=5.19, p=.001$; happy, $t(133)=4.28, p=.001$; and fear expressions, $t(133)=-2.29, p=.02$, but not for the expression of disgust, $p>.05$. The preferred social distance from Asian faces was wider for happy and neutral expressions, while participants indicated a preference for a wider separation from fearful Caucasian faces (Table 9).

\section{Table 9}

Descriptive Statistics [Mean (SD)] for Social Distance With Respect to Emotion and Race

$$
\text { Happy Fear Disgust }
$$

Neutral

\begin{tabular}{lllll}
\hline Asian & 3.67 & 3.42 & 4.46 & 4.67 \\
\hline Caucasian & 3.34 & 3.17 & 4.58 & 4.66
\end{tabular}


To explore the last significant interaction effect-mask, race, and emotion-we conducted paired $t$-tests for each emotional condition. Table 10 displays the results of these tests and Table 11 displays descriptive statistics of those conditions.

\section{Table 10}

Results of Paired t-tests for Masked and Unmasked Asian and Caucasian Faces

\begin{tabular}{|c|c|c|c|c|}
\hline Emotion & $\begin{array}{l}\text { Conditions } \\
\text { compared }\end{array}$ & Paired $t$ test results & Conditions & Paired $t$ test results \\
\hline Neutral & Asian M-UM & $t(133)=6.5, p=.001$ & $\begin{array}{l}\text { Caucasian M- } \\
\text { UM }\end{array}$ & $\mathrm{t}(133)=4.79, p=.001$ \\
\hline Happy & Asian M-UM & $\begin{array}{l}t(133)=4.84, p= \\
.001\end{array}$ & $\begin{array}{l}\text { Caucasian M- } \\
\text { UM }\end{array}$ & $t(133)=5.37, p=.001$ \\
\hline Fear & Asian M-UM & $\begin{array}{l}t(133)=7.22, p= \\
.001\end{array}$ & $\begin{array}{l}\text { Caucasian M- } \\
\text { UM }\end{array}$ & $t(133)=7.22, p=.001$ \\
\hline Disgust & Asian M-UM & $\begin{array}{l}t(133)=9.99, p= \\
.001\end{array}$ & $\begin{array}{l}\text { Caucasian M- } \\
\text { UM }\end{array}$ & $\begin{array}{l}t(133)=10.15, p= \\
.001\end{array}$ \\
\hline
\end{tabular}

\section{Table 11}

Descriptive Statistics [Mean (SD)] for Social Distance with Respect to Masked and Unmasked Asian and Caucasian Faces for Different Emotional Expressions

\begin{tabular}{lllll} 
& Neutral & Happy & Fear & Disgust \\
\hline Unmasked Asian & $4.1(1.99)$ & $3.8(2.15)$ & $5.07(1.81)$ & $5.28(1.82)$ \\
\hline Masked Asian & $3.24(1.73)$ & $3.04(1.65)$ & $3.84(1.66)$ & $4.06(1.78)$ \\
\hline Unmasked Caucasian & $3.39(1.62)$ & $3.74(1.99)$ & $4.99(1.77)$ & $5.34(1.83)$ \\
\hline Masked Caucasian & $3.78(1.99)$ & $2.89(1.56)$ & $4.16(1.67)$ & $3.98(1.66)$
\end{tabular}

The $t$-test results and descriptive statistics demonstrate that social distance changed as a function of emotion and mask wearing (Figure 8). Notably, the participants preferred to keep a long distance from unmasked faces, whether Asian or Caucasian. The stimuli showing fearful and disgusted faces sparked 
the longest social distance for both masked and unmasked faces. No significant race difference in social distance emerged for unmasked happy, fearful, or disgusted faces. However, the social distance for fearful or disgusted unmasked faces was significantly longer than that for neutral or happy unmasked faces. We also found no significant difference between masked disgusted Asian and Caucasian faces. Interestingly, the social distance for unmasked neutral Caucasian faces were shorter than the masked neutral Caucasian faces. Lastly, the social distance for masked fearful and neutral Caucasian faces was longer than the distance for the corresponding Asian faces.

\section{Discussion}

In this investigation, we conducted three experiments to explore the effects of mask wearing on face perception, emotion recognition, and social distance during the COVID-19 pandemic. The other race effect on these dependent variables was also investigated. The first experiment aimed to build an understanding of how interacting with masked faces might change face recognition. Accordingly, four conditions were used to compare face recognition: (a) seeing an unmasked face-recognizing the unmasked face, (b) seeing a masked face-recognizing the masked face, (c) seeing an unmasked facerecognizing the same face with a mask, and (d) seeing a masked face-recognizing that face without the mask.

\section{Face Perception}

\section{Mask Impact}

The experimental results indicated a significant mask impact on accuracy. The participants' performance was the best when they were asked to recognize an unmasked face that they had seen previously. Probably the faces were processed holistically in the unmasked conditions. Since the participants were familiar with holistic processing throughout their life, this strategy might have helped them recognize the unmasked faces accurately. Meanwhile, seeing and recognizing a masked face was the second condition that the participants could perform well. The participants were able to remember a masked face well if they had seen it with a mask beforehand. It might be expected that people perform better in recognizing faces that exactly match the sample conditions in the test phase. However, participants performance was the best when the faces were unmasked. On the other hand, their performance decreased when they first saw an unmasked face and then had to recognize that face with a mask. Presumably, the participants processed the unmasked sample faces holistically but found it difficult to match the whole face with a part of that face (e.g., eyes in a masked face). Interestingly, the participants' performance was better when matching a sample masked face with the unmasked form of that face in the testing stage. These results are similar to Noyes et al.'s (2021) and Freud et al.'s (2020) findings indicating that mask use impaired face recognition even in super-recognizers.

\section{Other-Race Impact}


Race was another critical factor in face recognition. The participants recognized Caucasian faces better than Asian faces. Since our subjects were Caucasian, Meissner and Brigham's (2001) ORB was also valid for our data. Moreover, we discovered a significant race and mask interaction effect. The participants' performance for the Caucasian unmasked sample-unmasked test condition was better than that for the masked sample-unmasked test and masked sample-masked test conditions. That said, the Caucasian unmasked-unmasked performance did not differ significantly from that for the unmasked samplemasked test condition. On the other hand, for Asian faces, participants showed the highest performance in the unmasked sample-unmasked test condition, while their performance for unmasked samplemasked test condition was lower than all the other conditions. Probably the participants could not process Asian faces effectively during sample presentation since they were not familiar with them. Conceivably, race changes the effect of a mask on face recognition. The most difficult condition for Caucasian faces was matching a sample masked face to the unmasked form of that face. In comparison, the most difficult condition for Asian faces was matching a sample unmasked face with a masked face. These results are consistent with Dhamecha et al.'s (2014) findings that hiding facial parts by accessories impaired face recognition ability, and recognition accuracy was better for faces from the same ethnicity. In a similar vein, Tanaka et al. (2004) and Michel et al. (2006) indicated that holistic face processing is affected by the other-race effect. Moreover, Hancock and Rhodes (2008) and Tanaka et al. (2004) proposed that facial identification is more efficient for faces that individuals experience more frequently but is less powerful for faces of other races that people have less experience with. Therefore, holistic face processing during the coding or testing phase involving Asian faces might have been less powerful than the holistic processing for Caucasian faces. Our results are consistent with Freud et al.'s (2020) study indicating that face recognition performance and holistic face processing decreased when participants were exposed to faces with masks during the COVID-19 pandemic.

\section{Emotion Recognition}

Experiment 2 was designed to investigate the effects of mask and race on emotion recognition accuracy. Listed from the most accurate to the least accurate, the participants recognized the emotional expressions under consideration as follows: happy, neutral, disgusted, and fearful.

\section{Mask Impact}

Mask wearing decreased recognition accuracy for all emotional expressions. Although the order of recognition accuracy for the various emotions did not change for masked faces, the accuracy for happy and neutral masked faces did not differ significantly It seems that the participants had difficulty in discriminating happy and neutral faces in the masked condition since happiness is mostly recognized via cues taken from the lower part of a person's face. This finding concurs with Bassili's (1979) demonstration that the recognition of happiness was reduced when the lower facial part was occluded with a rectangular piece of cardboard. Along the same lines, Carbon (2020) also indicated that wearing a face mask decreased the recognition accuracy of angry, disgusted, happy, and sad emotions but not neutral and fearful faces. Although our results concerning disgusted and happy expressions were 
consistent with the results of Carbon, mask use significantly decreased participants' recognition of the fear expression. Moreover, although disgust could be easily recognized on unmasked faces, recognition performance decreased dramatically when the faces were masked. This might be due to the closure of the cues around mouth those signal expression of disgust.

\section{Other-Race Impact}

Race was also a crucial factor in emotion recognition. The participants could recognize all Caucasian expressions better than the corresponding Asian expressions. However, the same recognition order was valid for both Caucasian and Asian expressions: happy, neutral, disgusted, and fearful (from the most to the least accurately recognized).

Although happiness was the most accurately recognized expression among all expressions for both unmasked Caucasian and Asian faces, performance decreased when the masked happy face was Asian. In contrast to Kret and De Gelder (2012) and Carbon (2020), fear was the least accurately recognized expression on both Caucasian and Asian faces. Interestingly, the participants could not recognize fear on Asian faces even when presented without a mask. It might be due to the task had included the seven options (six universal expressions and neutral). When participants were presented with fearful expressions, they might have responded as if they were surprised expressions in some trials.

\section{Social Distance}

In Experiment 3, we investigated the effects of mask, race, and emotions on preferred social distance with other people. Sorokowska and colleagues' (2017) data from 42 countries showed that average interpersonal social distance was $135.1 \mathrm{~cm}$, in general, and around $120 \mathrm{~cm}$ for the Turkish sample. In contrast, we found that the closest distance was 2.69 meters for masked Caucasian happy faces during the current COVID-19 pandemic and above 2.69 meters for all other stimulus types. The participants preferred to maintain a wider social distance with unmasked faces than masked faces. Conceivably, the pandemic has had a significant effect on social distance. Before the COVID-19 pandemic began, people tended to perceive facial masks as a sign of illness. However, the recent precautionary measures have called for everyone to use facial masks in social environments. Therefore, an unmasked person could be perceived as a potential virus carrier, causing people to avoid close contact with those not wearing facial masks. Thus, preferred social distance may have grown due to an increase in perceived risk of contamination, as indicated by Xie et al. (2020). Our findings are also consistent with Cartaud et al.'s (2020) results showing that facial mask wearing reduced social distancing. The participants preferred to keep away from unmasked faces, in general. The participants seemed to be convinced that a mask could protect them from exposure to the virus.

Mask use also interacted with emotions. The participants mostly tended to stay away from fearful and disgusted faces, both masked and unmasked. The preferred social distance for each emotional expression from farther to closer ranged in order from disgusted, fearful, and neutral to happy faces. The data were consistent with Ruggiero et al. (2017), who showed that perceiving positive emotions rather 
than negative emotions led to reducing social distance toward other people. The threatening nature of particular emotional expressions may also be crucial in social distancing. The stimuli featuring fearful and disgusted faces prompted the greatest social distance for both masked and unmasked faces. These two expressions signal an environmental threat via another person who conveys such information. A person signaling some kind of threat via a facial expression displaying a negative emotion may be interpreted as the threat being in close proximity to the signaler. Therefore, maintaining a large distance from that face can help the viewer cope with a potential threat. An expression evincing disgust may also result from experiencing poisoned food or illness. Therefore, a person showing this expression might potentially be ill or poisoned and could transmit illness by vomiting, sneezing, or coughing. In this event, observing a large distance can keep the viewer from being contaminated by any hazardous content.

In the case of emotions paired with unmasked faces, social distance was significantly greater for fearful and disgusted than neutral and happy faces. No significant race difference emerged regarding social distance for unmasked happy, fearful, and disgusted faces. There was also no significant difference between masked, disgusted Asian and Caucasian faces. However, the participants chose a larger social distance for masked fearful and neutral Caucasian faces than similar Asian faces. Presumably, the participants were better able to interpret the facial cues on the upper portion of each face when presented with Caucasian stimuli than they were able to with Asian faces due to the familiarity effect.

Race was another critical factor interacting with emotions. The participants preferred to establish a wider social distance from Asian faces than Caucasian faces. The preferred social distance from Asian faces was greater for happy and neutral expressions than the distance from Caucasian faces having the same expressions; however, it was wider for fearful Caucasian faces than for Asian fearful faces. They might have preferred a larger social distance from Asian neutral and happy faces in safe situations as a result of not being familiar with them or for reasons connected to the origins of the current pandemic. However, fear-related cues seemed to be better recognized on Caucasian faces and resulted in the participants' electing a larger social distance from Caucasian masked faces that exhibited fear.

Two reasons may underlie the participants' preference for larger social distance in the case of Asian faces. First, they might want to maintain more distance from unfamiliar-looking people about whom they did not have any information. The second reason might relate to the origins of the COVID-19 pandemic, which was first described in Wuhan, China. Although the COVID-19 virus has since that time spread worldwide, it was more prevalent in Eastern countries at the early stages of the pandemic. Therefore, Asian faces could be perceived as posing a higher risk of virus transmission during the data collection period.

Thus, familiarity and emotional expression seem to be critical factors in the participants' decision to adjust social distance.

\section{Conclusion}


In summary, face perception and social distancing have significantly changed over the course of the COVID-19 pandemic. Seeing and recognizing masked faces as well as trying to understand emotional expressions while adjusting social distance have become common challenges of daily life. Although humans process faces holistically, masked face perception may improve partial face processing ability in the near future. Moreover, although the other-race effect was evident for unmasked faces, this phenomenon has become more obvious since people from different races started wearing facial masks. Mask wearing decreased both face recognition and recognition of emotional expressions, negatively impacting communication among people from different ethnicities.

Although COVID-19 pandemic seems to decrease in most of the countries in the world recently, World Health Organization (WHO) explained that "it has not finished yet" and there are still possible serious scenarios for the future of the virus. In addition, there are other viruses such as influenza and rhinovirus, those can be transmitted through respiration and in the light of recent research on mask use (Howerd et al., 2021), it is clear that face masks protect against those viruses. Therefore, it seems that humanity will have to go on using face masks to have protection from respiratory transmitted diseases. One possible solution to future encounters with COVID-19-like viruses where humans must protect themselves with facial masks might be the production and use of transparent facial masks to improve face perception and communication skills with other people.

\section{Declarations}

\section{Author Note}

Evrim Gülbetekin (D) https://orcid.org/0000-0002-9359-3110

We have no conflicts of interest to disclose. This study's design and hypotheses were preregistered; see [https://osf.io/wxpr4/], [https://osf.io/cwvup/]

Correspondence concerning this article should be addressed to Evrim Gülbetekin, Department of Psychology, Faculty of Letters, Akdeniz University, Antalya, Turkey. Email: evrimg@akdeniz.edu.tr

\section{Abbreviations}

ORB = Own-race bias

$M=$ Masked

$\mathrm{UM}=$ Unmasked

\section{References}

Bassili, J. N. (1979). Emotion recognition: The role of facial movement and the relative importance of upper and lower areas of the face. Journal of Personality and Social Psychology, 37(11), 
Blazhenkova, O., Dogerlioglu-Demir, K. \& Booth, R.W. (2022). Masked emotions: Do face mask patterns and colors affect the recognition of emotions? Cognitive Research: Principles and Implications, 7, 33 https://doi.org/10.1186/s41235-022-00380-y

Carbon, C.-C. (2020). Wearing face masks strongly confuses counterparts in reading emotions. Frontiers in Psychology, 11, 2526. https://doi.org/10.3389/fpsyg.2020.566886

Carragher, D. J., \& Hancock, P. J. B. (2020). Surgical face masks impair human face matching performance for familiar and unfamiliar faces. Cognitive Research, 5, Article 59. https://doi.org/10.1186/s41235-020-00258-x

Cartaud A, Quesque F, \& Coello Y (2020) Wearing a face mask against Covid-19 results in a reduction of social distancing. PLOS ONE 15(12), e0243023. https://doi.org/10.1371/journal.pone.0243023

Cavazos, J. G., Noyes, E., \& O'Toole, A. J. (2018). Learning context and the other-race effect: Strategies for improving face recognition. Vision Research, 157, 169183. https://doi.org/10.1016/j.visres.2018.03.003

Chu, D. K, Akl, E. A, Duda, S, Solo, K, Yaacoub, S, Schünemann, H. J. (2020). Physical distancing, face masks, and eye protection to prevent person-to-person transmission of SARS-CoV-2 and COVID-19: A systematic review and meta-analysis. The Lancet, 395, 1973-1987.doi:10.1016/S0140-6736(20)31142-9 32497510

Conley, M. I., Dellarco, D. V., Rubien-Thomas, E., Cohen, A. O., Cervera, A., Tottenham, N., \& Casey, B. J. (2018). The racially diverse affective expression (RADIATE) face stimulus set. Psychiatry research, 270, 1059-1067. https://doi.org/10.1016/j.psychres.2018.04.066

Dal Ben, R. (2019). SHINE color and Lum_fun: A set of tools to control luminance of colored images (Version 0.2) [Computer software]. https://doi.org/10.17605/osf.io/auzjy

Dal Martello, M. F., \& Maloney, L. T. (2006). Where are kin recognition signals in the human face? Journal of Vision, (6), 1356-1366. https://doi.org/10.1167/6.12.2

Dhamecha, T. I., Singh, R., Vatsa, M., \& Kumar, A. (2014). Recognizing disguised faces: Human and machine evaluation. PLoS One, 9(7), e99212. https://doi.org/10.1371/journal.pone.0099212

Farah, M. J., Wilson, K. D., Drain, M., \& Tanaka, J. N. (1998). What is "special" about face perception? Psychological Review., 105, 482-498. https://doi.org/10.1037/0033-295X.105.3.482

Fischer, A. H., Gillebaart, M., Rotteveel, M., Becker, D., \& Vliek, M. (2012). Veiled emotions: The effect of covered faces on emotion perception and attitudes. Social Psychological and Personality Science, 33, 266-273. https://doi.org/10.1177/1948550611418534 
Freud, E., Stajduhar, A., Rosenbaum, R. S., Avidan, G., \&Ganel, T. (2020). The COVID-19 pandemic masks the way people perceive faces. Scientific Reports, 10, 22344. https://doi.org/10.1038/s41598-020-789869

Heron-Delaney, M., Anzures, G., Herbert, J. S., Quinn, P. C., Slater, A. M., Tanaka, J. W., Lee, K., \& Pascalis, O. (2011). Perceptual training prevents the emergence of the other race effect during infancy, PLOS ONE, 6(5). https://doi.org/10.1371/journal.pone.0019858

Hancock, K. J., \& Rhodes, G. (2008). Contact, configural coding and the other-race effect in face recognition. British Journal of Psychology, 99, 45-56. https://doi.org/10.1348/000712607x199981

Howard, J., Huang, A., Tufekçi, Z. et al. (2021) An evidence review of face masks against COVID-19. Proceedings of the National Academy of Sciences, 118, 4, e2014564118. doi: 10.1073/pnas.2014564118

Marini, M., Ansani, A., Paglieri, F. et al. (2021). The impact of facemasks on emotion recognition, trust attribution and re-identification. Sci Rep 11, 5577. https://doi.org/10.1038/s41598-021-84806-5

Megreya, A. M., \& Bindemann, M. (2009). Revisiting the processing of internal and external features of unfamiliar faces: The headscarf effect. Perception, 38(12), 1831-1848. https://doi.org/10.1068/p6385

Michel, C., Caldara, R., \& Rossion, B. (2006). Same-race faces are perceived more holistically than otherrace faces. Visual Cognition, 14, 55-73. https://doi.org/10.1080/13506280500158761

Noyes, E., Davis, J. P., Petrov, N., Gray, K. L. H., \& Ritchie, K. L. (2021). The effect of face masks and sunglasses on identity and expression recognition with super-recognizers and typical observers. Royal Society Open Science, 8, 201169. https://doi.org/10.1098/rsos.201169

Rezlescu, C., Susilo, T., Wilmer, J. B., \& Caramazza, A. (2017). The inversion, part-whole, and composite effects reflect distinct perceptual mechanisms with varied relationships to face recognition, Journal of Experimental Psychology: Human Perception and Performance, 43(12), 1961-1973. https://doi.org/10.1037/xhp0000400

Roberson, D., Kikutani, M., Döge, P., Whitaker, L., \& Majid, A. (2012). Shades of emotion: What the addition of sunglasses or masks to faces reveals about the development of facial expression processing. Cognition, 125(2), 195-206. DOI: 10.1016/j.cognition.2012.06.018

Ruggiero, G., Frassinetti, F., Coello, Y., Rapuano, M., di Cola, A,S., \& lachini, T. (2017). The effect of facial expressions on peripersonal and interpersonal spaces. Psychological Research, 81(6), 1232-1240. https://doi.org/10.1007/s00426-016-0806-x

Sorokowska, A., Sorokowski, P., Hilpert, P., Cantarero, K., Frackowiak, T., Ahmadi, K., Alghraibeh, A. M., Aryeetey, R., Bertoni, A., Bettache, K., Blumen, S., Błażejewska, M., Bortolini, T., Butovskaya, M., Castro, F. 
N., Cetinkaya, H., Cunha, D., David, D., David, O. A., ... Pierce, J. D. (2017). Preferred interpersonal distances: A global comparison. Journal of Cross-Cultural Psychology, 48(4), 577592. https://doi.org/10.1177/0022022117698039

Strohminger, N., Gray, K., Chituc, V., Heffner, J., Schein, C., \& Heagins, T. B. (2016). The MR2: A multi-racial, mega-resolution database of facial stimuli. Behavior Research Methods, 48(3), 1197-1204. https://doi.org/10.3758/s13428-015-0641-9

Tanaka, J. W., \& Farah, M. J. (1993). Parts and wholes in face recognition. Quarterly Journal of Experimental Psychology Section A, 46(2), 225-245. https://doi.org/10.1080/14640749308401045

Tanaka, J. W., Kiefer, M., \& Bukach, C. M. (2004). A holistic account of the own-race effect in face recognition: Evidence from a cross-cultural study. Cognition, 93, B1-B9. doi: 10.1016/j.cognition.2003.09.011.

Toseeb, U., Bryant, E. J., \& Keeble, D. R. T. (2014). The Muslim headscarf and face perception: "They all look the same, don't they?" PLoS One, 9(2): e84754. https://doi.org/10.1371/journal.pone.0084754

Welsch, R., Hecht, H., Chuang, L., \& von Castell, C. (2020). Interpersonal Distance in the SARS-CoV-2 Crisis. Human Factors, 62(7),1095-1101. doi:10.1177/0018720820956858

Wegrzyn, M., Vogt, M., Kireclioglu, B., Schneider, J., \& Kissler, J. (2017). Mapping the emotional face. How individual face parts contribute to successful emotion recognition. PLOS ONE, 12(5), e0177239. doi:10.1371/journal.pone.0177239

Yin, R. (1969). Looking at upside-down faces. Journal of Experimental Psychology, 81, 141145. http://dx.doi.org/10.1037/h0027474

Young, A. W., Hellawell, D., \& Hay, D. C. (1987). Configurational information in face perception. Perception, 16, 747-759. http://dx.doi .org/10.1068/p160747

\section{Figures}
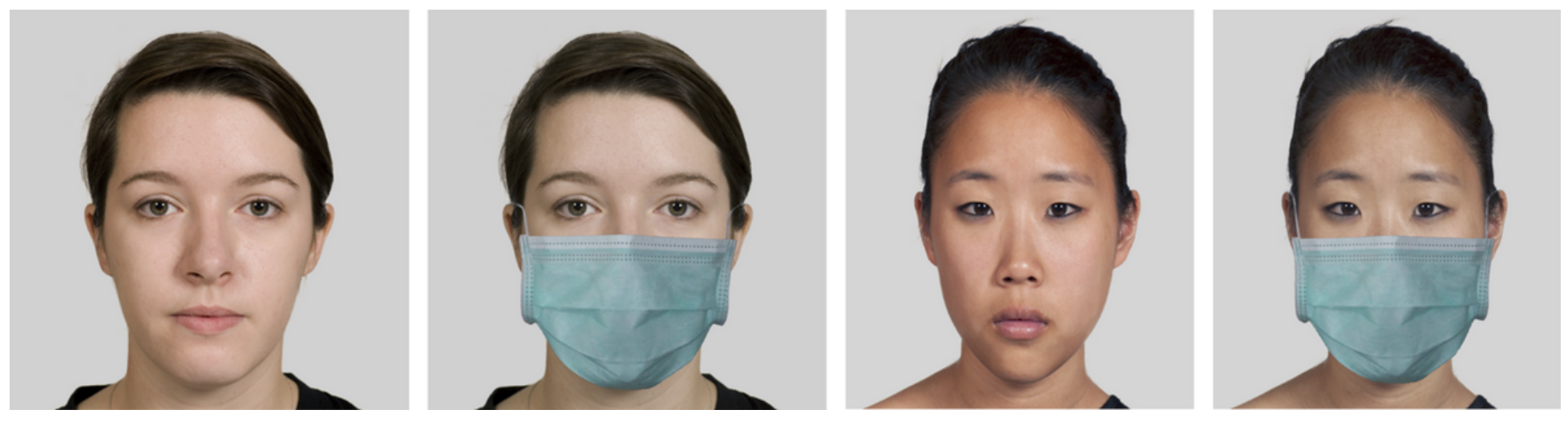

Figure 1 

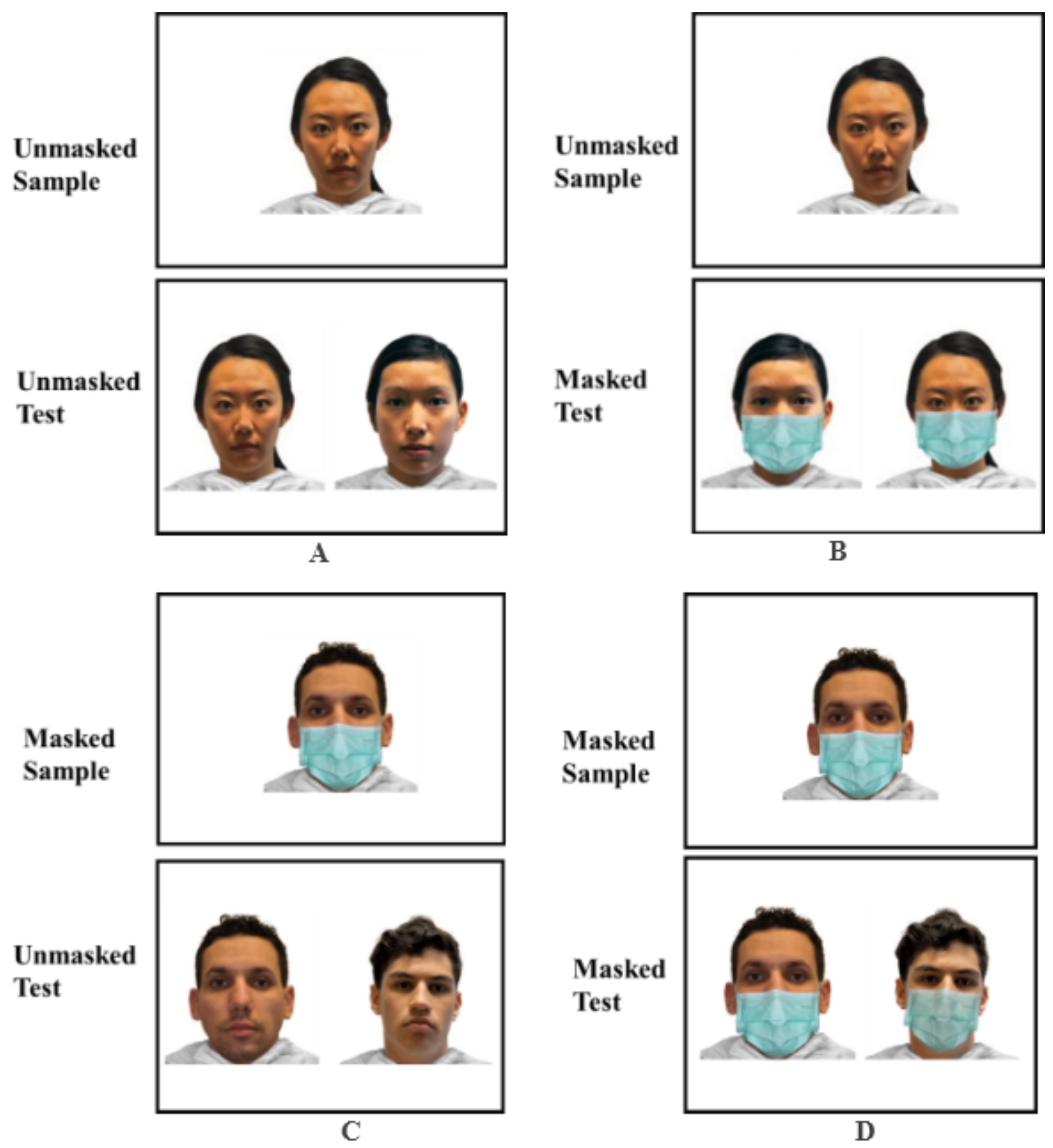

Figure 2

Experimental Conditions. Note. (A) An unmasked sample was presented, then unmasked faces were presented in the test phase. (B) An unmasked sample was presented, then masked faces were presented in the test phase. (C) A masked sample was presented, then unmasked faces were presented in the test phase. (D) A masked sample was presented, then masked faces were presented in the test phase. 


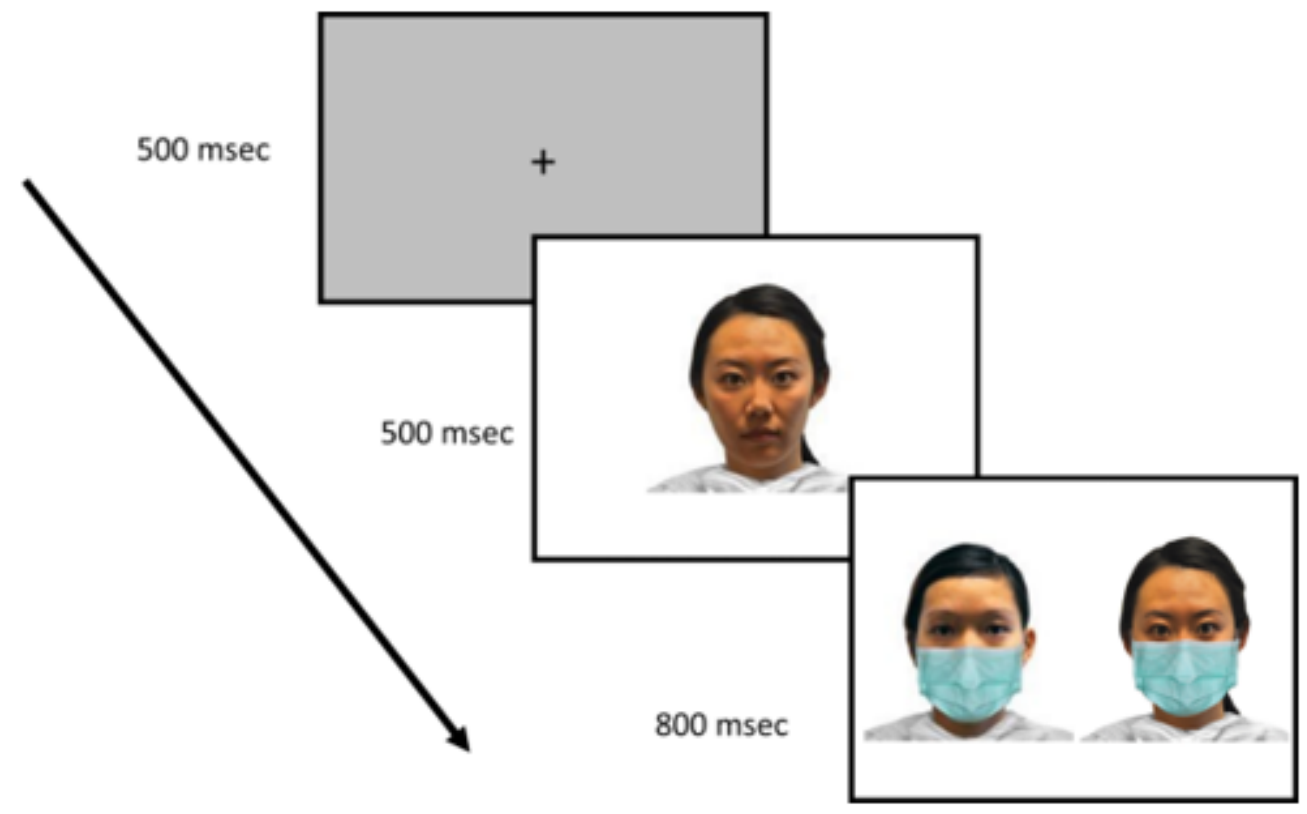

Figure 3

Experimental Procedure

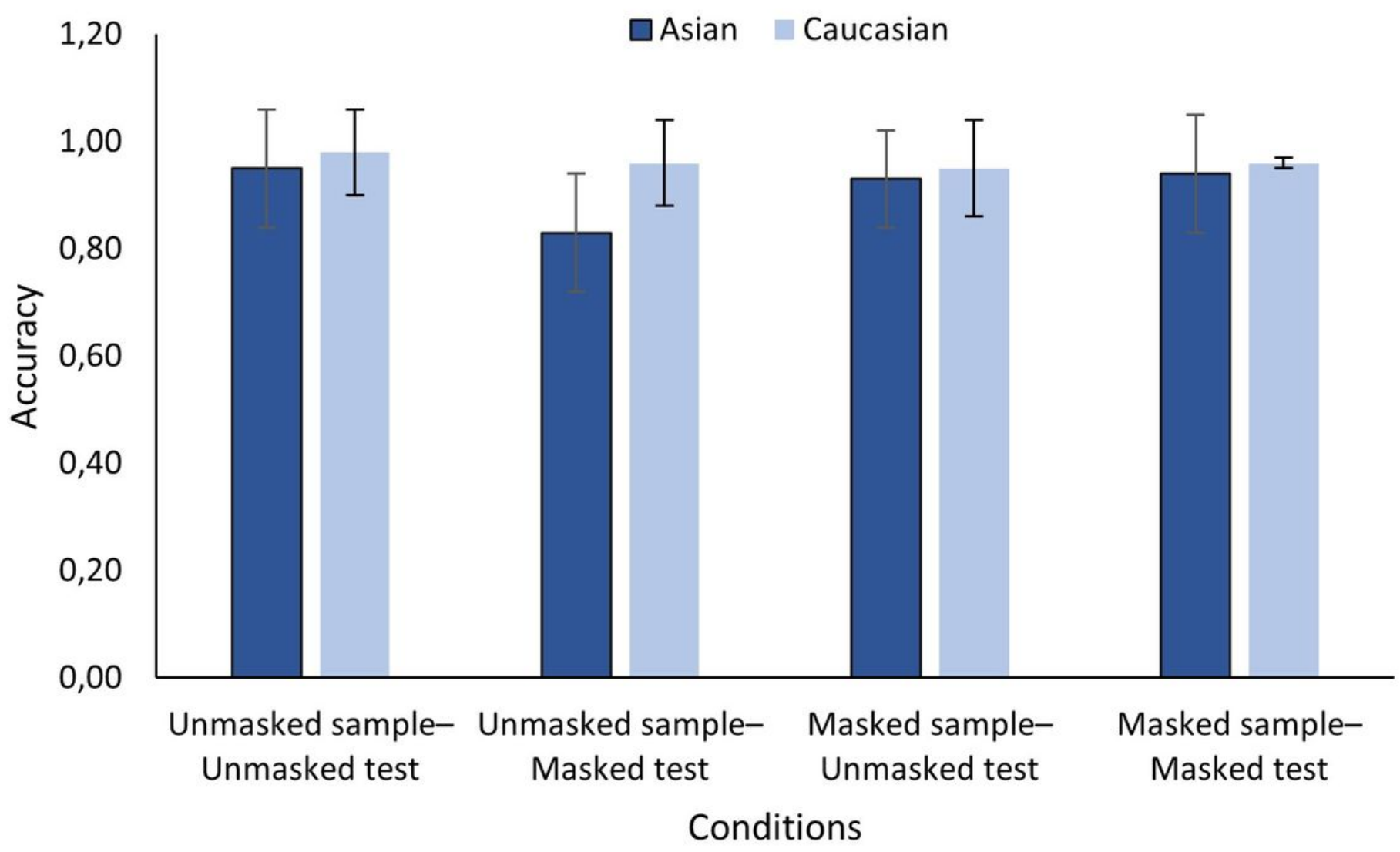

Figure 4 
Mean Accuracy for Race and Mask Conditions

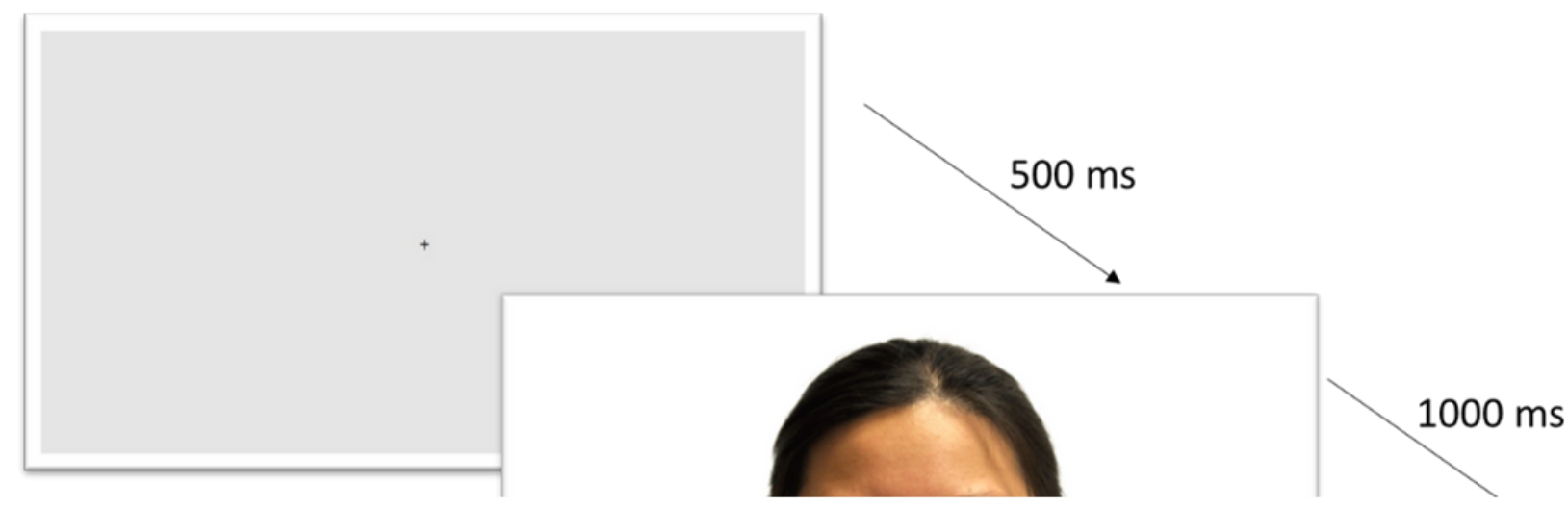

Figure 5

Experimental Procedure 


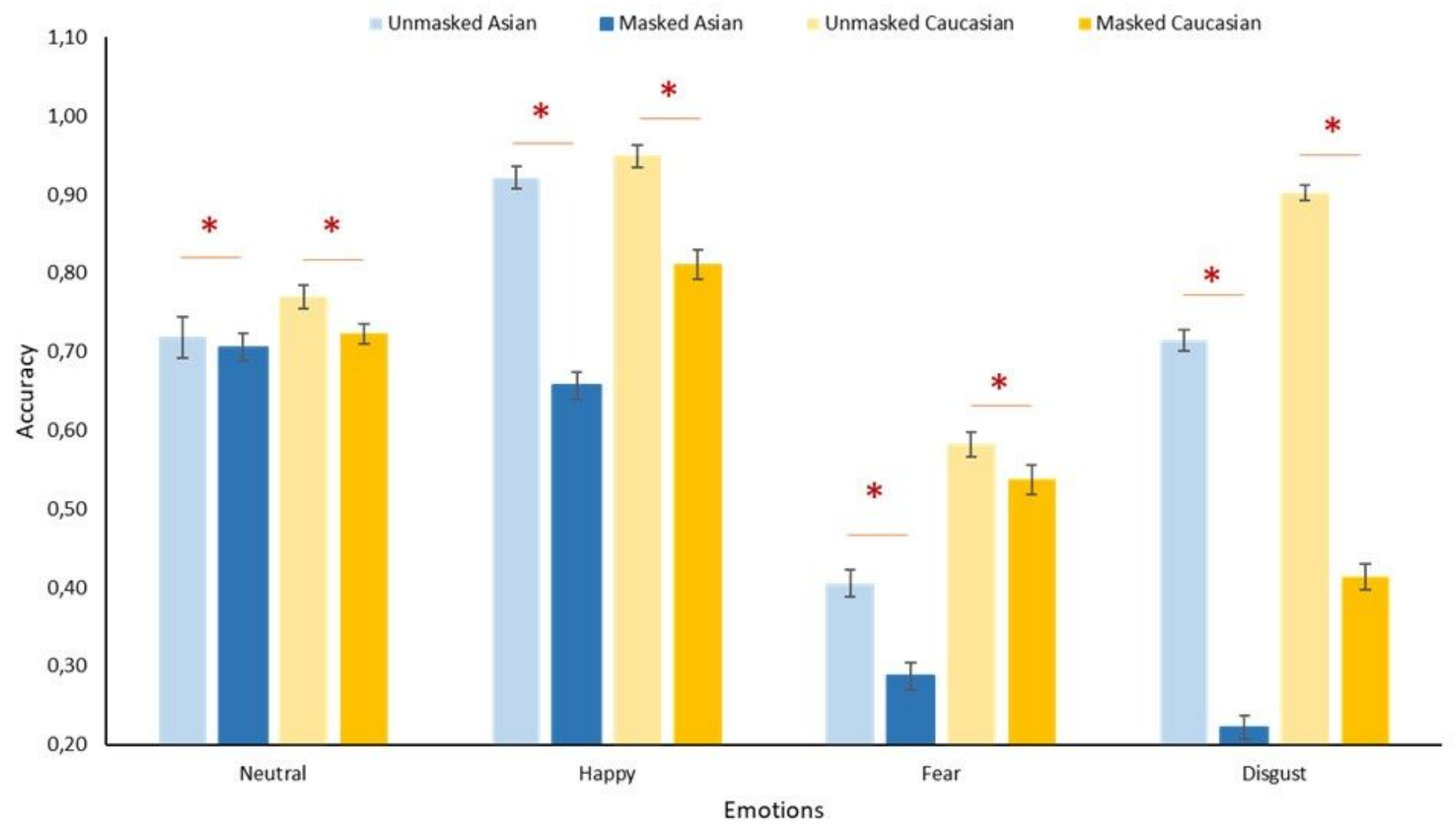

Figure 6

Mean Accuracy for Different Mask, Race, and Emotional Conditions 


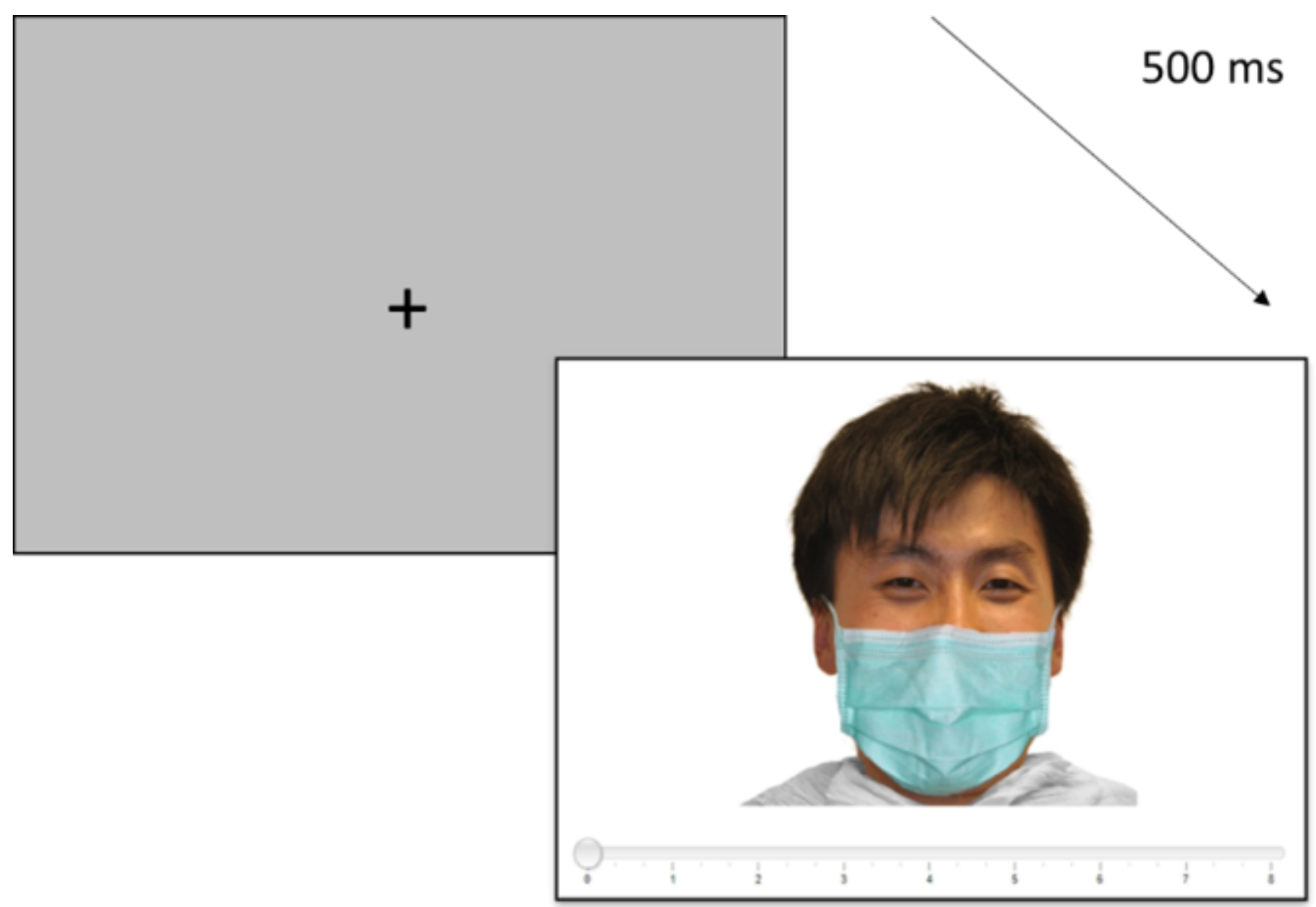

Figure 7

Experimental Procedure 


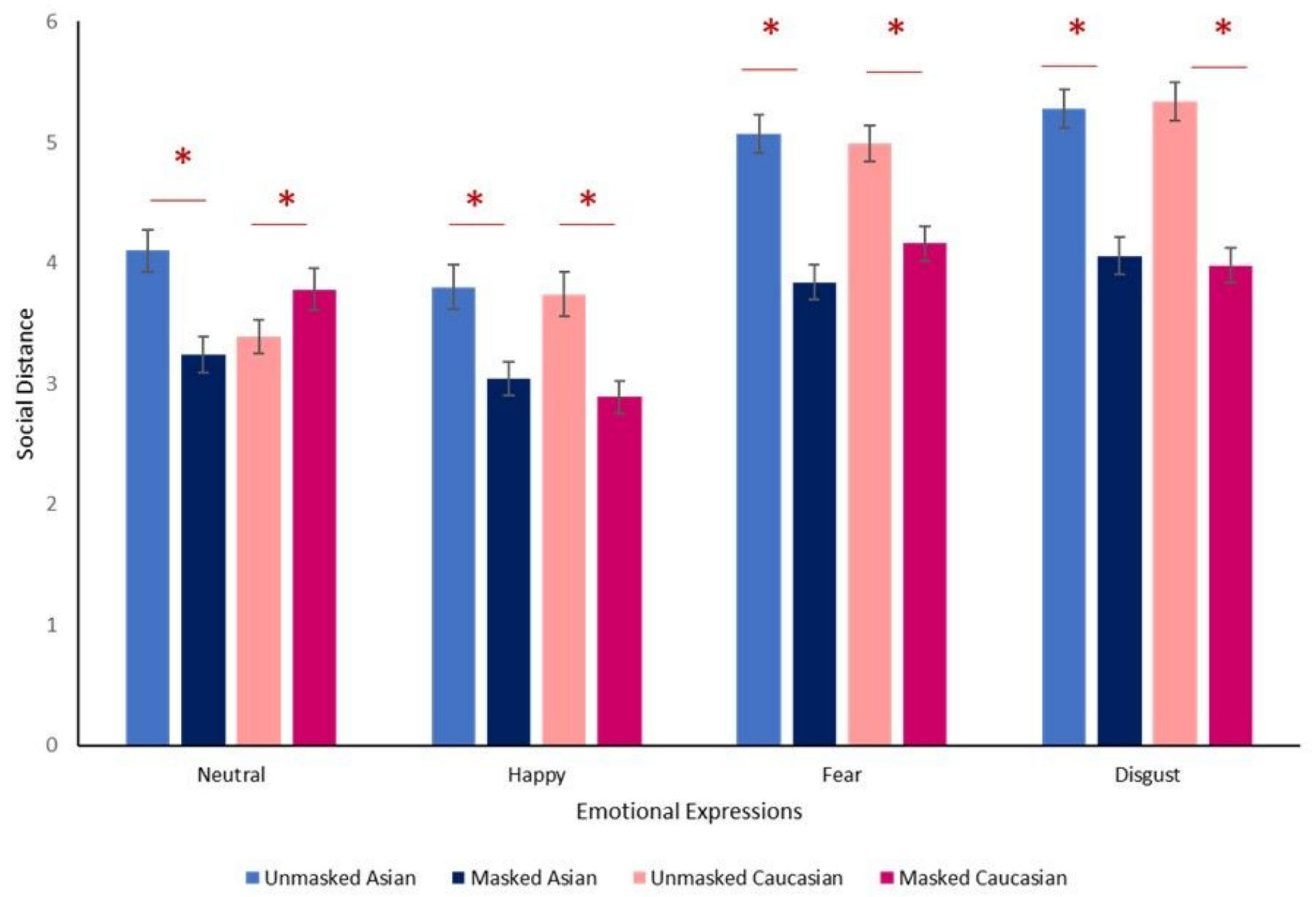

Figure 8

Participants' Preferred Social Distance with Respect to Emotional Expression and Race

\section{Supplementary Files}

This is a list of supplementary files associated with this preprint. Click to download.

- SuplementaryMaterials5.06.2022.docx 